\title{
RETICULATED VITREOUS CARBON: A USEFUL MATERIAL FOR CELL ADHESION AND TISSUE INVASION
}

\author{
Martina K. Pec ${ }^{1}$, Ricardo Reyes ${ }^{1}$, Esther Sánchez ${ }^{1}$, David Carballar ${ }^{2}$, Araceli Delgado ${ }^{1}$, Jesús Santamaría ${ }^{3}$, Manuel \\ Arruebo $^{3}$, and Carmen Evora ${ }^{1, *}$ \\ ${ }^{1}$ Department of Chemical Engineering and Pharmaceutical Technology, University of La Laguna, La Laguna, Spain \\ ${ }^{2}$ Pathological Anatomy Service, University Hospital Nuestra Señora de Candelaria, Santa Cruz de Tenerife, Spain \\ ${ }^{3}$ Department of Chemical Engineering, University of Zaragoza, Zaragoza, Spain
}

\begin{abstract}
Diverse carbon materials have been used for tissue engineering and clinical implant applications with varying success. In this study, commercially available reticulated vitreous carbon (RVC) foams were tested in vitro and in vivo for compatibility with primary cell adhesion and tissue repair. Pores sizes were determined as $279 \pm 98 \mu \mathrm{m}$. No hydroxyapatite deposition was detected after immersion of the foams in simulated body fluid. Nonetheless, RVC provided an excellent support for adhesion of mesenchymal stem cells (MSCs) as well as primary chondrocytes without any surface pre-treatment. Live cell quantification revealed neutral behaviour of the material with plastic adhered chondrocytes but moderate cytotoxicity with MSCs. Yet, rabbit implanted foams exhibited good integration in subcutaneous pockets and most importantly, total defect repair in bone. Probably due to the stiffness of the material, incompatibility with cartilage regeneration was found. Interestingly and in contrast to several other carbon materials, we observed a total lack of foreign body reactions. Our results and its outstanding porous interconnectivity and availability within a wide range of pore sizes convert RVC into an attractive candidate for tissue engineering applications in a variety of bone models and for ex vivo cell expansion for regenerative medical applications.
\end{abstract}

Keywords: Reticulated vitreous carbon, pore-size, porous interconnectivity, simulated body fluid, mesenchymal stroma cells, chondrocytes, cell adhesion, tissue compatibility, bone repair.

\footnotetext{
*Address for correspondence

Carmen Evora

Department of Chemical Engineering and Pharmaceutical Technology

University of La Laguna

Av. Astrofísico Francisco Sánchez, s/n

E-38200 La Laguna, Santa Cruz de Tenerife, Spain

Telephone Number: +34-922-318507

FAX Number: +34-922-318506

E-mail: cevora@ull.es
}

\section{Introduction}

Reticulated vitreous carbon (RVC) foam was first developed in the mid to late 1960 s as a thermally insulating, microporous glassy carbon electrode material. RVC foam is a strong, inert, electrically and thermally conductive, and corrosion resistant porous form of carbon with a low resistance to fluid flow. Due to these characteristics, the most widespread scientific use of RVC is in electrochemistry (Sumita, 1985; Friedrich et al., 2004; Rogulski et al., 2004). Additionally, RVC foams are characterized by an exceptionally high void volume, high surface area, and very high thermal resistance in nonoxidizing environments, which allows for heatsterilization and facilitates manipulation in biological applications.

A whole new area of applications for RVC opened up following the papers of Wickham et al. (1983) and later of Kent and Mutharasan (1992) who reported favourable properties of the material as a substrate for in vitro cell growth. The high surface area, high porosity, regular structure, and low fluid flow resistance convert the RVC foams into attractive candidates for scaffolds for tissue regeneration. In the tantalum biomaterial Hedrocel ${ }^{\mathrm{TM}} \mathrm{RVC}$ was used as a vitreous carbon core with layers of crystalline tantalum deposited by means of chemical vapour infiltration (Zardiackas et al., 2001). This rendered the constructs with a high resistance to weight loading but at the same time the honeycomb-like, inter-connected porous structure of its carbon skeleton favoured tissue invasion. Since then, the material has been used as hip joint and other orthopaedic implants and subjected to various clinical studies (Wigfield et al., 2003; Komarasamy et al., 2006; Mulier et al., 2006). However $\mathrm{RVC}$ is, by no means, the only carbon material the use of which as a clinical implant has been advocated. The most important examples of competing materials are discussed next.

Proplast I, a polymer consisting of vitreous carbon fibres and polytetrafluoroethylene (Teflon $\left.{ }^{\circledR}\right)$ and its next generation variant Proplast II, a composite of Teflon $\mathbb{R}$ polymer and alumina, were reported among others as eye implants in rabbits (Proplast I) and as subperiosteal implants in anophthalmic patients exhibiting no significant foreign body response or extrusion (Barber et al., 1980; Shah et al., 1995). However, several clinical case reports with temporomandibular joint Teflon ${ }^{\circledR}$-Proplast implants reported exuberant foreign body reactions, implant displacements and fractures. The authors insist in their 
recommendation that these implants should be removed in all patients (Lypka et al., 2007; Abramowicz et al., 2008).

The application of various different carbon fibre based implants was reported in clinical tendon and osteochondral lesions, generally describing good results during the follow-up phase except for a carbon fibre rod, implanted in a rabbit osteochondral defect, which was found to even prevent subchondral bone restoration and re-establishment of the normal osteochondral junction (Parsons et al., 1989; Visuri et al., 1991; Nicholson et al., 1998; Qiu et al., 2002).

Aoki et al. (2009) developed carbon fibre sheets with a nano- to microscale pore structure out of electrospun polymic solutions, pre-adsorbed with rhBMP-2 (recombinant human bone morphogenetic protein 2) and implanted them in dorsal muscle pockets and critical size ileum bone defects. Although the authors reported no severe inflammatory reaction with the control implants, they found a complete foreign-body granuloma 6 weeks after implantation of the control sheets. Moreover, defect repair only occurred in the presence of BMP-2.

Commercial Bicarbon ${ }^{\mathrm{TM}}$ bileaflet valves (Sorin Biomedica Cardio, Saluggia, Italy), composed of two pyrolytic carbon leaflets hinged on a titanium alloy housing coated with a thin carbon layer (Carbofilm ${ }^{\mathrm{TM}}$ ) have been evaluated extensively in multicentre, long-term follow-up clinical studies (Borman et al., 1998; Misawa et al., 2007). The clinical data point out excellent features associated with a low incidence of valve-related complications.

Turgut et al. (2008) developed a series of carbon foams from combining pitch with various additives under variable temperature and pressure release conditions, reaching a maximum total porosity of $80.6 \%$ with pore sizes oscillating between 37.6 and $82.1 \mu \mathrm{m}$. They observed loose connective tissue invasion and formation of fibrous capsules around the materials implanted in subcutaneous pockets and monocortical bone defects in rats.

The conventional surgical techniques to boost tissue regeneration sometimes do not lead to the desired results, especially in cases of large, critical size injuries. To date, regenerative medicine as an upcoming, diversified field for investigation from bench to bedside includes among others direct growth factor therapy, implantation of in vitro expanded cell populations, and gene therapy by delivering the desired genetic information through various vehicles, as may be transfected cells. Hence, porous matrices may not only help to support the structure in critical size defects and guide the regeneration process but also serve as delivery systems for regeneration-promoting molecules and perhaps most importantly as a support for cell attachment, proliferation and differentiation. Thus, the development of adequate scaffolds is a key issue in tissue engineering.

In this paper, Reticulated Vitreous Carbon (RVC) foams with highly interconnected pores of honeycomb structure were studied for their application as potential scaffolds. Cytotoxicity and biocompatibility were evaluated in vitro with bone marrow derived mesenchymal stroma cells (MSCs) and primary chondrocytes and in vivo in a rabbit model.

\section{Materials and Methods}

\section{Material characterization}

Duocel® RVC foam 100 PPI, 3 \% relative density, was purchased from ERG Materials and Aerospace Corp. (Oakland, CA, USA) and mechanized as $3 \times 3 \mathrm{~mm}$ and a 6 x $10 \mathrm{~mm}$ cylinders for in vitro and in vivo experiments, respectively.

Physicochemical characterization of the samples was carried out by SEM-EDX, X-ray diffraction (XRD) and $\mathrm{X}$-ray photoelectron analysis (XPS).

The XRD spectra were recorded at room temperature on a Rigaku (Tokyo. Japan) D/Max diffractometer with a rotary anode using a $\mathrm{CuK} \alpha$ source $(\gamma=1.5418 \AA)$ operating at $40 \mathrm{kV}$ and $80 \mathrm{~mA}$. Data were collected from 5 to $80^{\circ}$ with a $0.03^{\circ}$ step and a step scan of $1 \mathrm{~s} /$ step. Data were processed with JADE (Christchurch, New Zealand) software.

The samples were also examined by scanning electron microscopy (SEM) in a JEOL (Tokyo, Japan) JSM-6400 instrument operating at $3-20 \mathrm{kV}$.

$\mathrm{X}$-ray photoelectron analysis (XPS) was performed with an Axis Ultra DLD (Kratos Analytical, Manchester, UK). The cylindrical pieces were mounted on a sample rod, placed in the pre-treatment chamber of the spectrometer and then evacuated at room temperature. A monochromatized $\mathrm{Al} \mathrm{K} \alpha$ source $(1486.6 \mathrm{eV})$ run at $15 \mathrm{kV}$ and $10 \mathrm{~mA}$ was used. For the individual peak regions, a pass energy of $20 \mathrm{eV}$ was used. Survey spectrum was measured at $160 \mathrm{eV}$ pass energy. Analyses of the peaks were performed with the software provided by the manufacturer, using a weighted sum of Lorentzian and Gaussian component curves after background subtraction.

\section{Bone-like apatite layer deposition}

To evaluate a possible bone-like hydroxyapatite layer formation on the RVC cylinder surfaces, five samples were incubated statically in simulated body fluid (SBF), prepared as solution 9 in the work of Kokubo et al. (1990) at $37^{\circ} \mathrm{C}$ during 1 month.

\section{Cells}

Rat bone marrow stromal cell isolation

Bone marrow mesenchymal stroma cells (MSCs) were obtained from 6 weeks old male Sprague-Dawley rats by centrifugal isolation as described by Dobson et al. (1999). Whole bone marrow was pooled and resuspended in Dulbecco's Minimal Essential medium (DMEM) with 4.5 g/l glucose, supplemented with $20 \%$ foetal bovine serum (FBS), $50 \mathrm{UI} / \mathrm{ml}$ penicillin, $50 \mu \mathrm{g} / \mathrm{ml}$ streptomycin, and $200 \mathrm{mM}$ stabilised L-glutamine and seeded on cell culture plastic. Cells were cultured at $37^{\circ} \mathrm{C}$ and $5 \% \mathrm{CO}_{2}$. After three days, cultures were rinsed carefully with calcium and magnesium free phosphate buffered saline (PBS) to remove non-adhered cells and fresh culture medium was added. Cells were detached when confluent and frozen for later experimental use.

\section{Rabbit chondrocyte isolation and culture}

Cartilage slices from rabbit knee joints were aseptically chopped off with a scalpel. Slices were transferred to 
centrifuge tubes and incubated with $0.05 \%$ trypsin/EDTA for $15 \mathrm{~min}$ in a shaking water bath, at $37^{\circ} \mathrm{C}$ and $50 \mathrm{rpm}$. After a centrifugation step at $500 \mathrm{~g}$ for $5 \mathrm{~min}$ the pellet was resuspended in a collagenase IV solution (Sigma, St. Louis, MO, USA) at an enzyme concentration of $1 \mathrm{mg} / \mathrm{ml}$ in DMEM with $4.5 \mathrm{~g} / 1$ glucose, supplemented with $10 \%$ FBS and incubated for $150 \mathrm{~min}$ as before. After short precipitation of the remaining cartilage the supernatant was transferred to another tube, centrifuged at $500 \mathrm{~g}$ for $5 \mathrm{~min}$ and the resuspended pellet seeded in DMEM supplemented with $10 \%$ FBS, $110 \mathrm{mg} / \mathrm{l}$ sodium-pyruvate (BioWhittaker, Walkersville, MD, USA), and penicillin-streptomycin and stabilised L-glutamine as above on cell culture plastic. Confluent cells were detached and frozen for later experimental use.

Unless otherwise specified, all cell culture material was from PAA (Linz, Austria) and culture plastic from Nunc (Langenselbold, Germany).

\section{Cytotoxicity assay}

$7.99 \times 10^{2}$ freshly thawed rat MSCs and $1.44 \times 10^{3}$ rabbit chondrocytes, both in the first subculture passage (p1), were seeded in quadruplicates in wells of a 96-well plate in their respective culture medium. Cells were allowed to precipitate at $37^{\circ} \mathrm{C}$ for $1 \mathrm{~h}$ and then an autoclaved implant was added with sterile forceps to each well. Medium was replaced on the 4th day of culture. After 6 (chondrocytes) and 7 days (MSCs) implants were removed and relative cell numbers with respect to control wells without implants were quantified with the colorimetric XTT tetrazolium assay (Roche, Madrid, Spain) following the manufacturer's instructions. Briefly, the tetrazolium salt XTT is metabolized by mitochondrial dehydrogenases of viable cells to a formazan dye. Numerical values of colour densities were obtained in a Synergy HT plate reader (BioTek, Winooski, VT, USA) at a wavelength of $490 \mathrm{~nm} /$ reference $690 \mathrm{~nm}$.

\section{Scaffold cell loading and labelling}

The autoclaved scaffolds were submerged in PBS to reduce liquid rejection by electrostatic loading. The excess fluid around the scaffold was aspirated with a Pasteur pipette. Then $15 \mu \mathrm{l}$ of a rabbit chondrocyte or rat MSC suspension in culture medium at a concentration of $1 \times 10^{6} \mathrm{cells} / \mathrm{ml}$ were loaded onto each scaffold by pipetting. The constructs were incubated for $2 \mathrm{~h}$ in the $\mathrm{CO}_{2}$ incubator at $37^{\circ} \mathrm{C}$. They were then transferred to culture wells coated with bovine serum albumin (BSA) (1\% in PBS; Sigma) and the respective culture medium was added. Medium was changed every 3-4 days.

After 2 weeks, scaffolds were rinsed with PBS, fixed with formaldehyde ( $4 \%$ in PBS) overnight at $4^{\circ} \mathrm{C}$, rinsed with PBS, and permeabilized with $0.2 \%$ Triton X-100 in PBS for $30 \mathrm{~min}$ at room temperature, followed by another rinse with PBS. Then cells were labelled at room temperature under light protection for 15 min with a propidium iodide solution (Sigma) in PBS at a concentration of $4 \mu \mathrm{g} / \mathrm{ml}$ and again rinsed with PBS. Visual inspection and fluorescent photography where carried out with a Zeiss (Jena, Germany) Axiovert 40 CFL inverted microscope and a Canon (Tokyo, Japan) PowerShot A620 digital camera.

\section{Animal experiments}

All the experiments were carried out in conformity with the E.C. Guideline on the Protection of Animals Used for Experimental and Other Scientific Purposes.

\section{In vivo biocompatibility assays}

The surgical procedure for osteochondral and bone defects comprised a vertical external parapatellar incision in the right knees of male New Zealand rabbits (3-4 kg) followed by a dislocation of the patellar tendon and quadriceps to allow access to the femoral condyles. After scaffold insertion into the damaged femur, the patella and the patellar tendon were reduced.

Osteochondral defects $(3 \mathrm{~mm}$ in diameter and $3 \mathrm{~mm}$ in depth) were created with the knee maximally flexed, making a drill-hole in the weight-bearing area of the medial femoral condyle. The scaffold $(3 \times 3 \mathrm{~mm})$ was then carefully inserted into the defect by press-fit fixation.

For bone defect setting, a hole was made with a $6 \mathrm{~mm}$ dental burr in the intercondylar space reaching the medullar cavity.

Subcutaneous scaffold implantation was carried out by making an incision on the dorsal side of the rabbit and inserting a single scaffold into the pocket.

Sampling time points for all tissues were 2, 6 and 12 weeks. The animals were allowed free movement.

\section{Histological analysis}

Defect bearing including femurs and subcutaneous scaffolds (two specimens per sampling time point) were prepared for histological evaluation. Samples were fixed in $10 \%$ formaldehyde in phosphate buffer ( $\mathrm{pH} 7.4)$. Femurs were then decalcified in $10 \%$ formic acid solution, dehydrated in a graded series of ethanol and embedded in Paraplast. Microtome (Shandon Finesse 325, Thermo Fisher Scientific Inc., Waltham, MA, USA) sections of 3$5 \mu \mathrm{m}$ thickness were prepared throughout the defect/ scaffold site.

Sections were deparaffinised in xylol, rehydrated in a graded series of ethanol and stained with HaematoxylinEritrosin (H-Er), Cleveland-Rucker-Wolfe (CRW) and Masson-Goldner Trichrome for structure characterization.

\section{Immunohistochemical analysis}

Sections were deparaffinised and rehydrated in Trisbuffered saline (TBS) (pH 7.4, 0.1 M), which was also used for all further incubation and wash steps. The sections underwent antigen retrieval in citrate buffer $(\mathrm{pH} 6,10 \mathrm{mM})$ at $65^{\circ} \mathrm{C}$ for 20 minutes and were then blocked with FBS at $2 \%$ in a TBS-Triton $\mathrm{X}-100$ solution. The indirect immunohistochemical procedure was carried out by incubating the sections overnight at $4^{\circ} \mathrm{C}$ with the following antisera: osteocalcin antiserum (1/100) and collagen typeI antiserum (1/100) (Millipore, Barcelona, Spain), and after a rinse step, with a biotin-SP-conjugated $\mathrm{F}(\mathrm{ab}$ ') fragment donkey anti-rabbit antibody (1/200) (Millipore) for $60 \mathrm{~min}$. After rinsing, sections were incubated with peroxidase- 

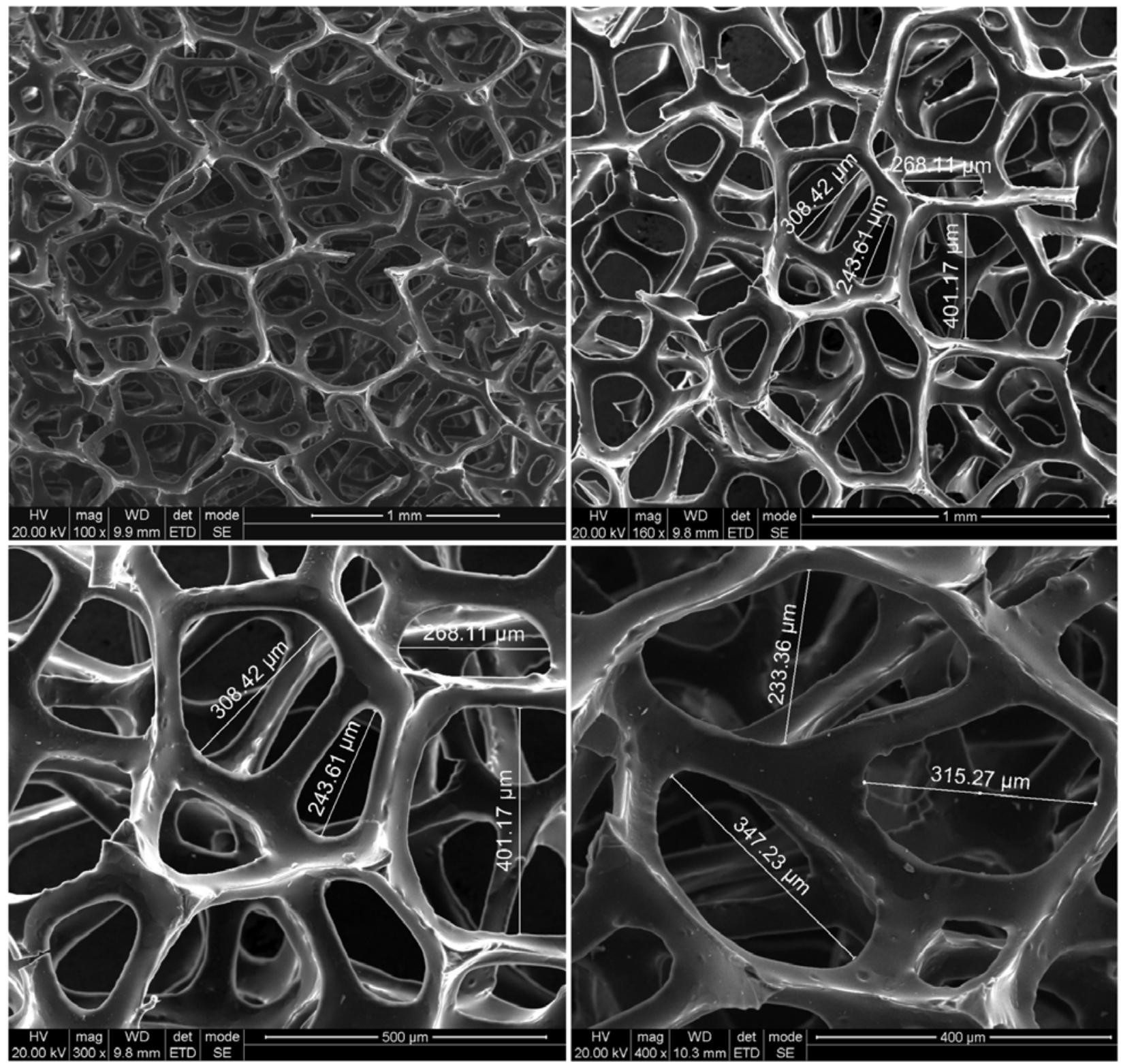

Fig. 1. SEM micrographs showing at different magnifications the structure and the pore size of the RVC foams.

conjugated streptavidin (1/300) (Millipore) for $60 \mathrm{~min}$. Peroxidase activity was revealed in Tris- $\mathrm{HCl}$ buffer $(\mathrm{pH}$ 7.6, $0.05 \mathrm{M})$ containing $0.04 \%$ of 4-chloro-1-naphtol (Sigma) and $0.01 \%$ of hydrogen peroxide. Specificity of immunolabelling was controlled by replacing the specific antiserum by normal serum.

Sections were analyzed using a LEICA DM 4000B light microscope (Leica Microsystems, Wetzlar, Germany) coupled to a digital LEICA DFC 300FX camera.

\section{Results}

\section{Physical-chemical characterization}

Pore sizes, determined by SEM, oscillated around $279 \pm$ $98 \mu \mathrm{m}(\mathrm{N}=40)$. Morphology and pore sizes of the open cell foams are shown in Fig. 1.

The chemical composition (element/C ratio) of the surface of non-treated RVC foams and of foams after 1 month of incubation in SBF, obtained by XPS is shown in Tables 1 and 2. Table 1 summarizes the binding energy (BE) corresponding to the peak assignments and shows the percentage of each element present on the surface of non-treated RVC. The presence of less than 1 at $\%$ of $\mathrm{Ca}$ and $\mathrm{P}$ on the scaffold surfaces after one month of immersion in SBF could be attributed to a superficial adsorption of fluid components. The increase of $\mathrm{N}$ and $\mathrm{Na}$ atomic concentrations can also be assigned to the SBF components. These results allow ruling out the formation of hydroxyapatite in significant amounts. XRD and SEM data further support this conclusion.

Moreover, the SEM images (Fig. 1) did not show any visible changes in surface morphology and no formation of hydroxyapatite crystals was observed. In addition, XRD patterns for the samples in SBF did not reveal new reflections for the SBF immersed samples and definitely did not show the characteristic hydroxyapatite diffraction peak (Fig. 2) which should be located around 30-34 degrees. 
Table 1. Atomic composition of the reticulated vitreous carbon foam obtained by XPS

\begin{tabular}{|c|c|c|}
\hline Element & BE (eV) & \% atomic \\
\hline C 1s & 284.9 & 89.0 \\
\hline O 1s & 532.6 & 9.8 \\
\hline Si $2 p$ & 101.7 & 0.4 \\
\hline N 1s & 399.9 & 0.8 \\
\hline
\end{tabular}

Table 2. Chemical composition (element/C ratio) obtained by XPS for the reticulated vitreous carbon before and after SBF incubation.

\begin{tabular}{|c|c|c|c|c|c|c|c|}
\hline \multirow{3}{*}{ 它 } & \multirow{2}{*}{ Sample } & \multicolumn{6}{|c|}{ Superficial Ratio (Element/C) } \\
\hline & & $\mathbf{O} / \mathbf{C}$ & $\mathrm{Na} / \mathrm{C}$ & $\mathrm{Ca} / \mathrm{C}$ & $\mathbf{P} / \mathbf{C}$ & $\mathrm{N} / \mathrm{C}$ & $\mathrm{Si} / \mathrm{C}$ \\
\hline & Cylinder & 0.11 & - & - & - & 0.009 & 0.005 \\
\hline \multirow{2}{*}{ 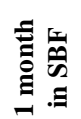 } & Pulverized & 0.16 & 0.001 & 0.001 & 0.001 & 0.035 & 0.031 \\
\hline & Cylinder & 0.15 & 0.005 & 0.003 & 0.004 & 0.051 & 0.002 \\
\hline
\end{tabular}

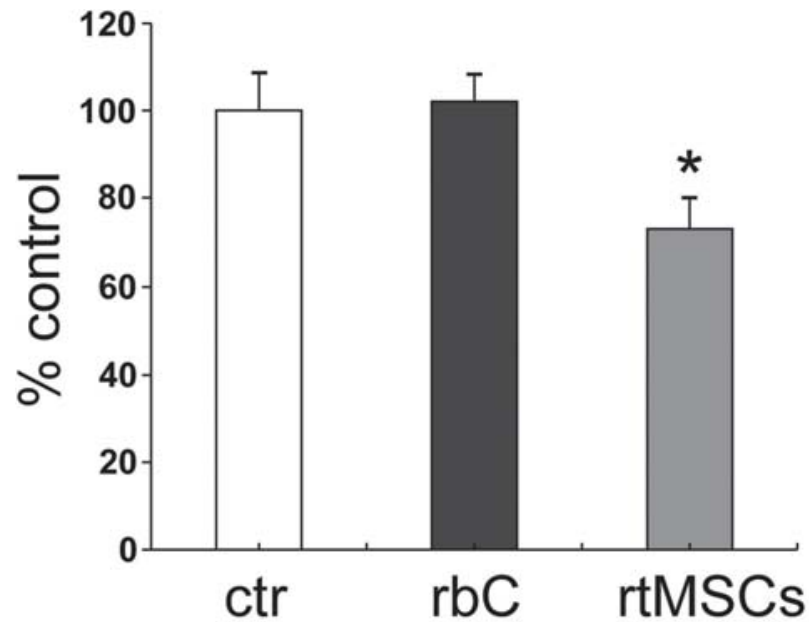

Fig. 3. Cytotoxicity of RVC material in primary chondrocyte and MSC 2D cultures. While rabbit chondrocytes (rbC) were not affected, rat MSCs (rtMSCs) suffered significant cytotoxicity $(* p<0.05)$ compared to untreated controls (ctr). Metabolic activity of cells was assessed by XTT assay (measures of optical density at $490 \mathrm{~nm}$ ). Data are expressed as mean percentage \pm SD of controls which were considered 100 $\%(\mathrm{~N}=3)$.

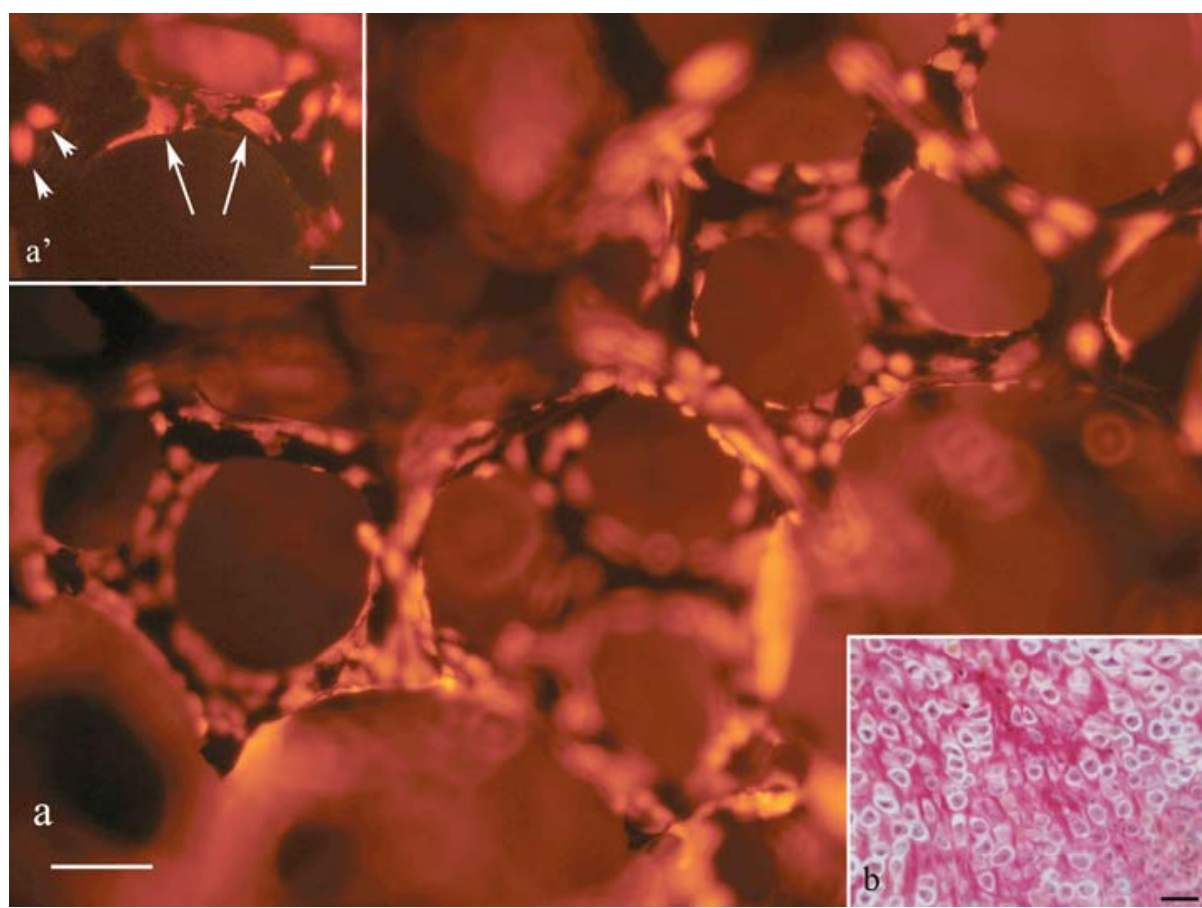

Fig. 4. Inverted microscopy image of propidium iodide stained primary rabbit chondrocytes in RVC scaffolds. Round or partially spread (arrow heads but also fully spread chondrocytes (arrows) (both a') were found adhered to the RCV fibres, whereas chondrocytes embedded within the extracellular cartilage matrix exhibit a totally round phenotype (b, Masson Trichrome staining). Scale bars: $\mathbf{a}=10 \mu \mathrm{m} ; \mathbf{a}, \mathbf{b}=20 \mu \mathrm{m}$. 


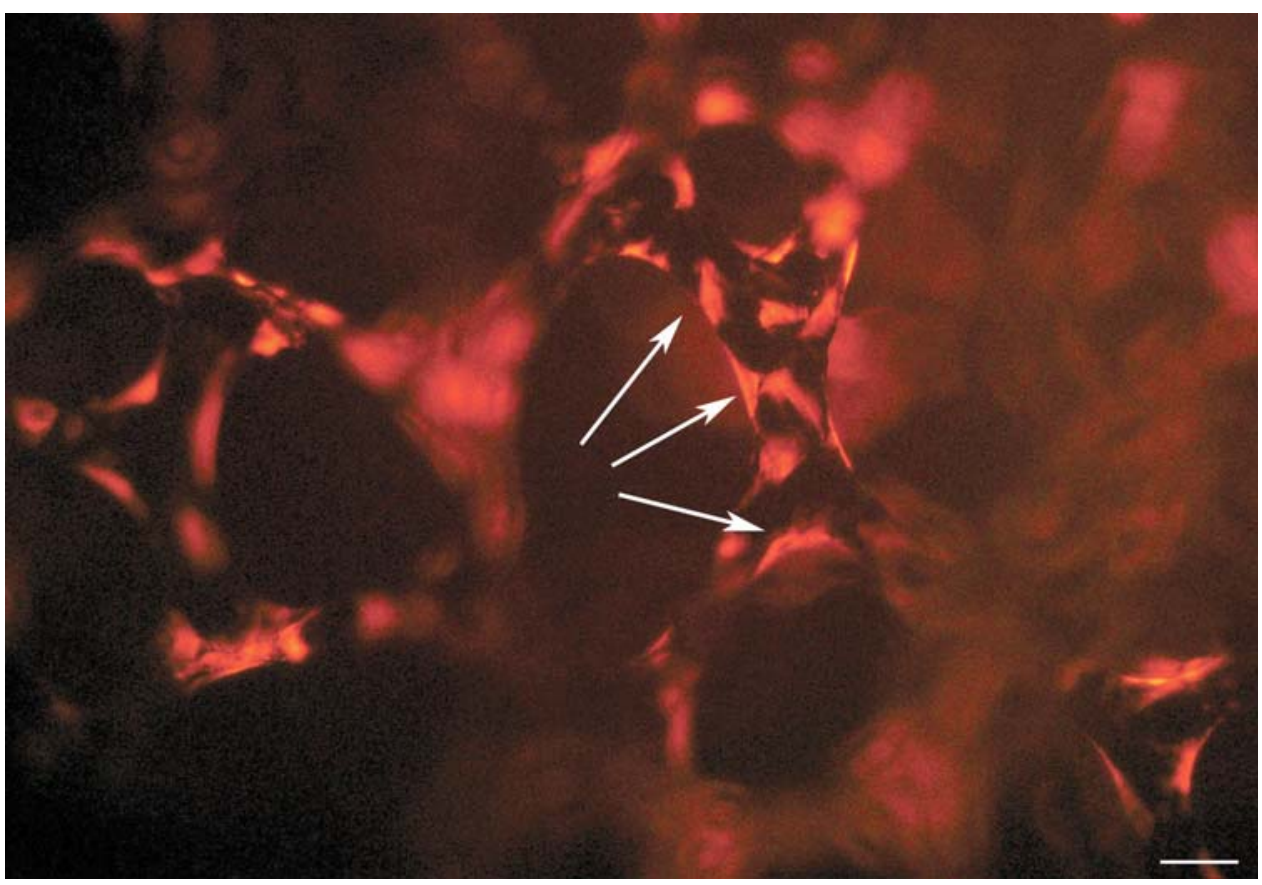

Fig. 5. Inverted microscopy image of propidium iodide stained primary rat MSCs in RVC scaffolds. MSCs were observed as a dense population of mainly fully spread cells (arrows) on the RCV fibres. Scale bar: $10 \mu \mathrm{m}$.
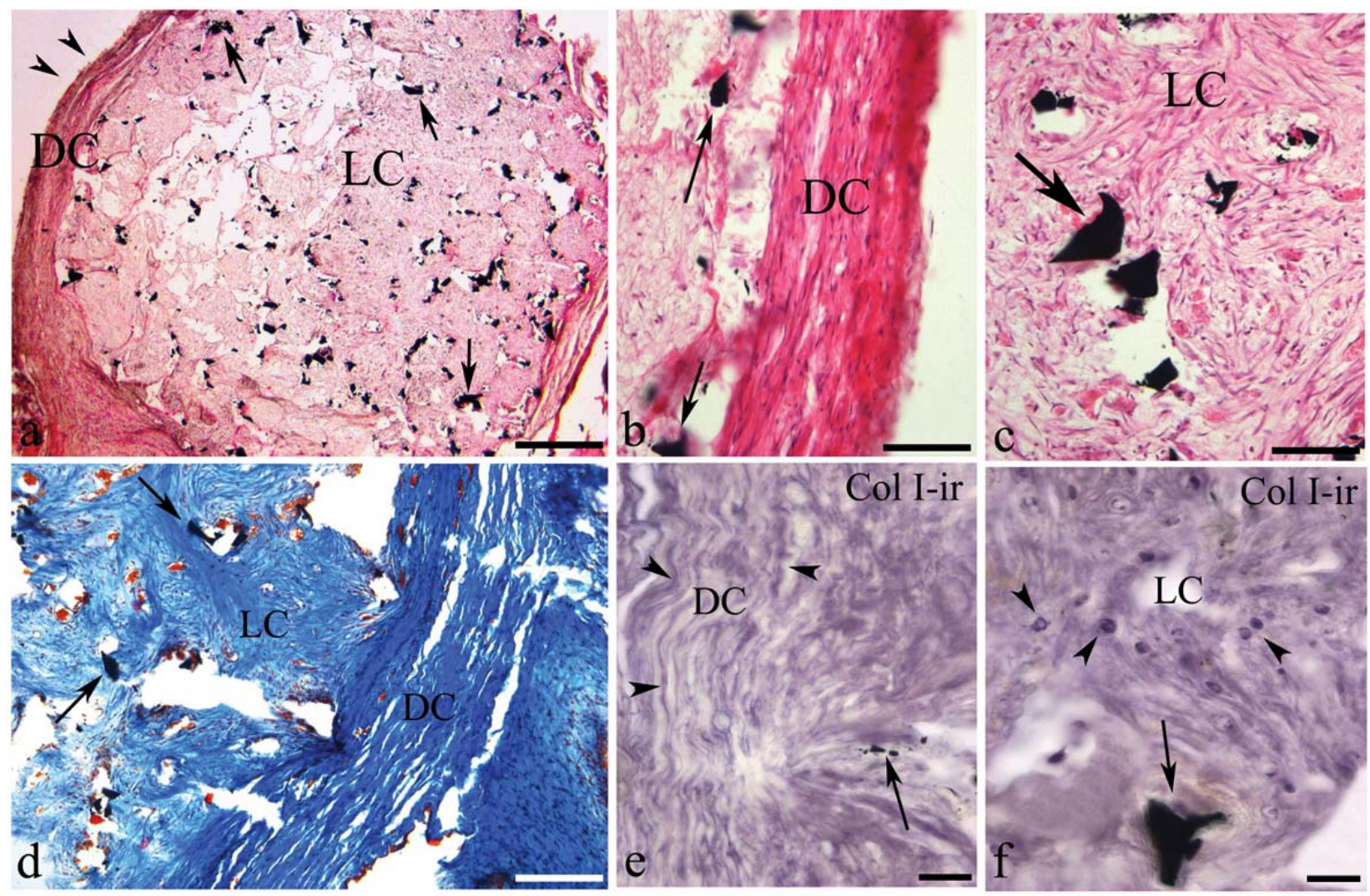

Fig. 6. Subcutaneous pocket. Representative images of rabbit skin with subcutaneously implanted RVC scaffolds at three sampling time points. (a) Horizontal section at two weeks post-implantation showing the subcutaneous scaffold filled with loose connective tissue and blood vessels and surrounded by a capsule (arrowheads) of dense connective tissue. Scaffold fragments (arrows) are associated with the voids left from the implant. (b,c) Details at high magnification of the external capsule of dense connective tissue (b) and the loose connective tissue within the scaffold structure (arrows) (c). The holes correspond to the spaces formerly occupied by the implant. (d-f) Horizontal sections from specimen assessed at twelve weeks post-implantation. (d) Histochemical staining for collagen (blue staining) (e,f) Immunohistochemical staining of collagen type I, scaffold structures (arrows), collagen fibres (e), and fibroblasts (f) (arrowheads). Scale bars: $\mathrm{a}=100 \mu \mathrm{m} ; \mathrm{b}, \mathrm{c}=50 \mu \mathrm{m} ; \mathrm{d}=65 \mu \mathrm{m} ; \mathrm{e}, \mathrm{f}=25 \mu \mathrm{m}$. LC: loose connective tissue, DC: dense connective tissue. 


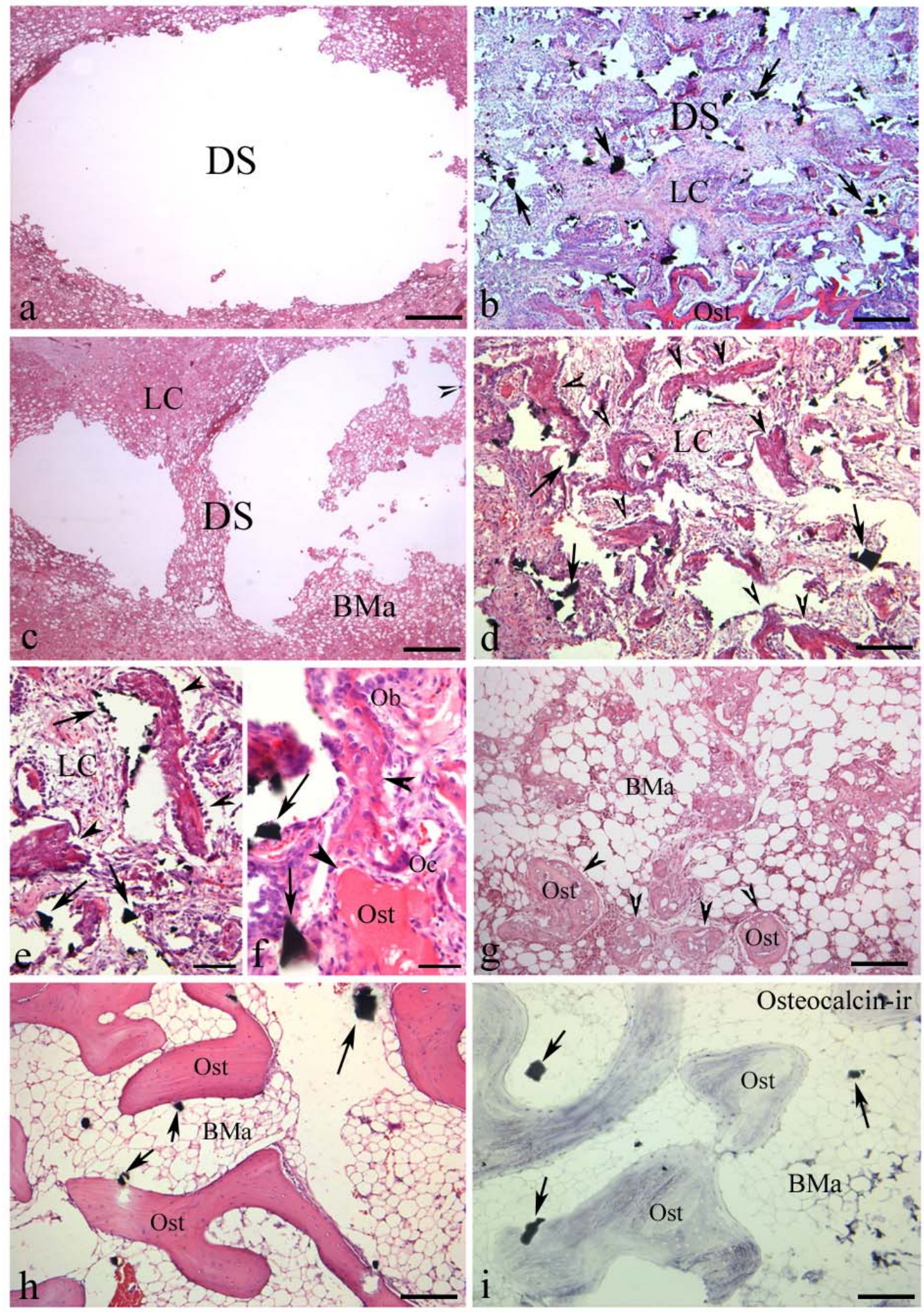

Fig. 7. Bone defect. Representative microphotographs of rabbit femurs from control animals (a, c, g) and RVC scaffold implanted animals (b, d, e, f, h, i) at three sampling time points. (a) Horizontal section of the empty defect site of a control animal at two weeks post-surgery. (b) Horizontal section at two weeks post-implantation showing the defect site filled with loose connective tissue and osteoid (Ost) in the defect margins. (c) Horizontal section of the defect site of a control specimen at six weeks post-surgery, being partially filled with loose connective tissue and bone marrow elements. (d) Horizontal section of an implanted defect site at six weeks post-implantation containing many ossification foci (arrowheads) associated with the scaffold fragments (arrows). (e,f) High magnification details of the previous image. Scaffold fragments (arrows) were found to be associated with the empty space left from the implant (b, d, e, f). (g) Horizontal section of a control specimen at twelve weeks post-surgery exhibiting ossification process and the formation of immature bone (arrowheads) within the defect site. (h) Horizontal section at twelve weeks post-implantation with mature bone trabeculae (arrowheads) within the defect site associated with scaffold fragments (arrows). (a-h): H-Er. (i) Osteocalcin-immunoreactivity in RVC scaffold implanted animals at twelve weeks post-implantation demonstrates the presence of mineralized bone trabeculae. Scale bars: a,b,c,d = $100 \mu \mathrm{m}$; e $=50 \mu \mathrm{m} ; \mathrm{f}=25 \mu \mathrm{m} ; \mathrm{g}, \mathrm{h}, \mathrm{i}=80 \mu \mathrm{m}$. DS: defect site, LC: loose connective tissue, BMa: bone marrow, Ost: osteoid, Ob: osteoblast, Oc: osteoclast. 


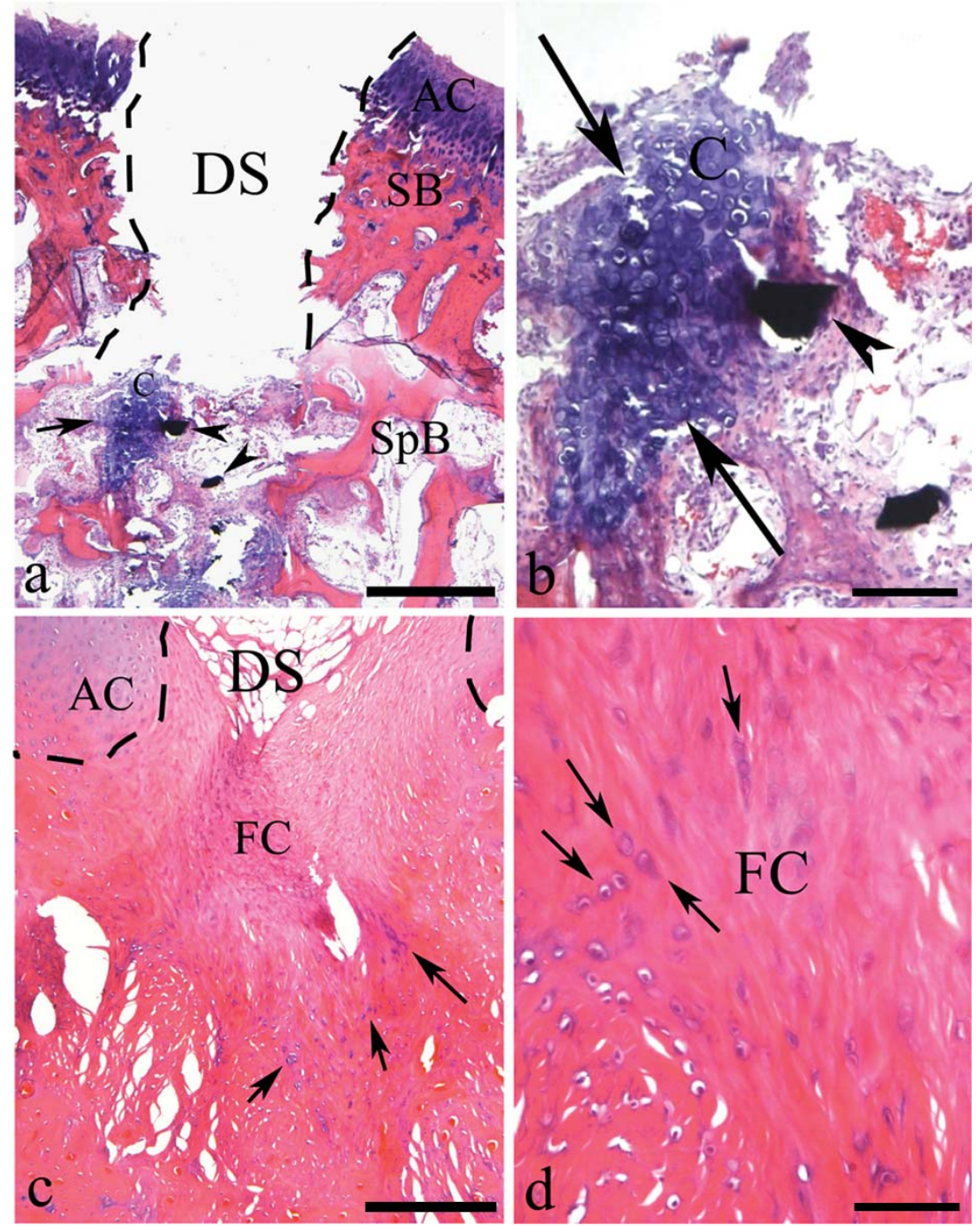

Fig. 8. Osteochondral defect. Representative microphotographs of RVC scaffold implanted rabbit femurs from three sampling time points. (a) Horizontal section from specimen at two weeks post-implantation without any sign of defect-repair. Neocartilage (C) (arrows) was found associated with a scaffold fragment (arrowheads) beneath the defect site. (b) Detail at high magnification demonstrating the tight association of ectopic cartilage (arrows) with the scaffold fragment (arrowheads). (c) Horizontal sections from specimen at twelve weeks post-implantation. The defect site is filled with a plug of fibrocartilage (FC), a dense connective tissue with cartilage elements associated (arrows). No scaffold fragments were detected within the defect site (d) Detail of the fibrocartilage structure at high magnification. AC: articular cartilage, C: cartilage, DS: defect site, FC: fibrocartilage, SB: subchondral bone, SpB: spongy bone. Scale bars: a, c $=$ $200 \mu \mathrm{m} ; \mathrm{b}, \mathrm{d}=50 \mu \mathrm{m}$.

\section{Cell culture evaluation \\ Cytotoxicity}

Plastic cultured (2 dimensional) rat MSCs suffered some cytotoxicity in the presence of the carbon foams, resulting in cell densities of around $70 \%$ compared to controls. Rabbit chondrocyte were not affected at all under equivalent conditions (Fig. 3).

\section{Cell cultures in scaffolds}

Although excess culture medium of both cell suspensions was found to pour out at the bottom of the scaffolds during loading manipulation, dense populations of rabbit chondrocytes as well as rat MSCs were discovered adhered to the fibres of the RCV foams. Round, partially spread and also fully spread chondrocytes were observed (Fig. $\left.4 a, a^{\prime}\right)$. Despite of the observed slight growth inhibition in 2D cultures, lots of rat MSCs adhered perfectly well to the RCV structures and most frequently exhibited a fully spread phenotype (Fig. 5).

\section{Histological evaluation}

From two weeks on (first sampling time point) good compatibility and integration were observed in the subcutaneous and the bone defect site but not in the osteochondral defects, where the scaffolds were found beneath the implantation site. Remarkably, not even the slightest sign of inflammation or foreign body reaction could be detected. Scaffolds were found to be fragmented but not degraded.

\section{Subcutaneous pocket}

The results obtained from the subcutaneously implanted scaffolds were very much alike at all three sampling time points, being completely integrated into the tissue structure from two weeks on. The scaffolds were surrounded by a capsule of dense connective tissue (Fig. 6a,b). Invasion of loose connective tissue and blood vessels of different sizes were observed (Fig. 6c). Histochemistry (Fig. 6d) and immunohistochemical staining for collagen type-I (Fig. 6e) revealed a normal structure and composition of the connective tissue from two weeks on.

\section{Bone defect}

Two weeks post-implantation, the defect sites still appeared empty in the control animals (Fig. 7a) whereas the defect sites with RVC scaffolds were completely filled with connective tissue (Fig. 7b). Osteoid formation was observed in the marginal zones of the defect sites where RVC scaffolds had been implanted (Fig. 7b).

At six weeks, the defect sites of the control animals appeared partially filled with loose connective tissue and bone marrow (Fig. 7c). Defects of RVC implanted animals 


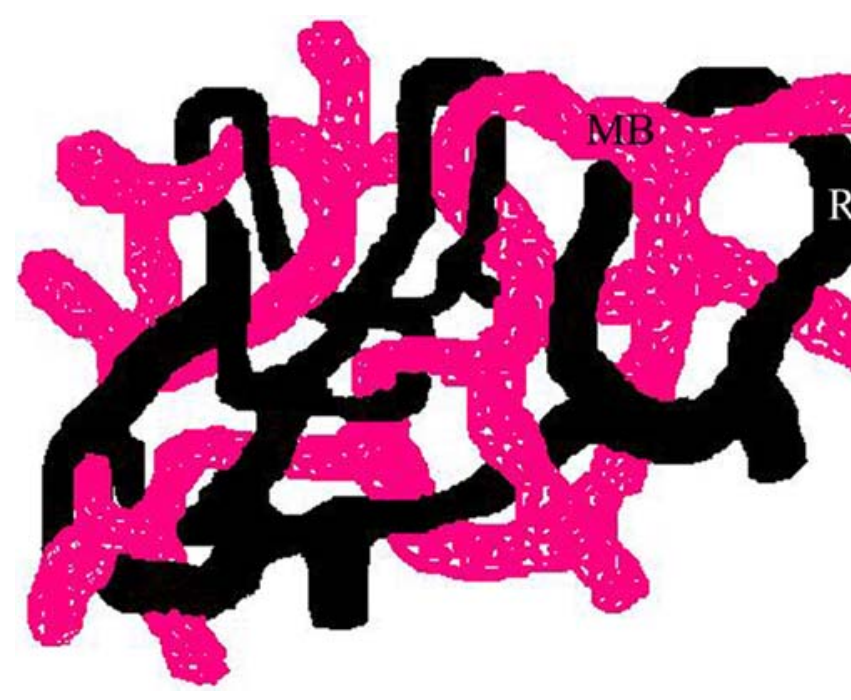

Fig. 9. Schematic drawing showing the proposed hypothetical structure, in which the scaffold is integrated into the structure of mature bone by the end of the regeneration process. MB: Mineralized bone, RVC: Reticulated vitreous carbon.

exhibited several active intramembranous ossification foci immersed in a mixed matrix of loose connective tissue containing bone marrow mesenchymal cells (Fig. 7d,e,f).

Finally, at twelve weeks, several ossification foci where observed in the defects of control animals (Fig. 7g). However, the RVC implanted defects had been completely regenerated with signs of ossification and mineralization equivalent to mature bone (Fig. $7 \mathrm{~h}$ ). There were mineral bone trabeculae, confirmed by osteocalcinimmunoreactivity (Fig. 7i). Scaffold fragments were found to be integrated into the newly formed tissue structure at all three sampling time points.

\section{Osteochondral defect}

No signs of repair could be detected at the defect site two weeks post-implantation. Ectopic neocartilage formation associated with the scaffold fragments was found outside the site of injury (Fig. 8a,b). At six and twelve weeks, the defect sites were filled with fibrocartilage, a dense connective tissue with cartilage elements. The implantation sites of the six and twelve week samples presented no visible differences (Fig. 8c,d). There were no scaffold fragments located within the defect sites at any of the sampling time points. No differences were found compared with control animals with empty osteochondral defects at the different time points (data not shown). However, unexpectedly, we did not find any sign of inflammation or foreign body reaction.

\section{Discussion}

Reticulated vitreous carbon (RVC), a material especially known in scientific, electrochemical application, has been characterized in the skeletal system and in connective tissue, in order to evaluate its properties with respect to tissue engineering. A variety of different carbon based materials has been previously described in ophthalmic (Barber et al. 1980, Wickham et al. 1983) and bone applications (Turgut et al., 2008; Aoki et al., 2009). Some have even reached clinical use (Borman et al., 1998; Lypka et al., 2007; Misawa et al., 2007; Abramowicz et al., 2008).

In this work, the implantation of Duocel ${ }^{\circledR}$ RVC foam scaffolds with pores oscillating between $200-400 \mu \mathrm{m}$ in rabbit bone defects and subcutaneous pockets revealed good integration into the surrounding tissue. Similarly as with the carbon foams developed by Turgut et al. (2008), loose connective tissue invasion and the formation of a fibrous capsule around the subcutaneous implants were observed. In contrast to the mentioned work, we did not find any capsule formation around our bone RVC implants and most importantly observed complete defect regeneration twelve weeks post-implantation with the nondegradable scaffold material being incorporated into the newly formed bone. The foams from Turgut et al. (2008) exhibited porosities of up to $80 \%$, initially a promising value to warrant mass transport throughout the implant. However, the pore size of less than $100 \mu \mathrm{m}$ most likely presented a limit for bone and vessel formation, which would explain the contrasting results in bone integration compared to our work.

Probably due to the brittle nature of the RVC material, sample processing resulted in implant fragmentation, evident in our histological specimens. The fragments were mainly located in direct association with tissue lagoons, which had been obviously filled with scaffold material before histological processing. Such fragment-lagoon associations were observed in loose connective tissue, typically in the subcutaneous implantation sites at all three analyzed time points, but also in the bone defects at two and six weeks post-implantation where loose connective tissue had formed as well. A few scaffold fragments were found in association with, but not penetrating, the bone trabeculae (Fig. 6e,f), most likely for similar reasons. Twelve weeks after implantation, most of the connective tissue from the early bone regeneration phase had been replaced by bone marrow. The implant fragments were located within the bone marrow around the newly formed bone trabeculae (compare our hypothetic drawing scheme Fig. 9), suggesting that the material firstly favours soft tissue invasion and formation and secondly, provides enough space for the development of mature bone matrix. 
Importantly, the empty control defects revealed that the Duocel ${ }^{\circledR}$ RVC foams provide an excellent instrument to accelerate bone defect repair leading to a much faster and total regeneration. Twelve weeks after defect setting the control damages had still been not completely regenerated while the implant filled defects had.

SBF has been and is still extensively used to predict bioactivity and especially bone bonding capacity of implant materials through the analysis of apatite formation on their surface (Kokubo et al., 1990). However, Bohner and Lemaitre (2009) successfully questioned this paradigm and referenced examples of well-characterized materials that show extensive bone bonding in vivo but do not allow for apatite formation in SBF, as for example $\beta$-tricalcium phosphate $(\beta$-TCP). On the other hand, materials like calcium silicate hydrate (CSH) and dicalcium phosphate dehydrate (DCPD) which are covered by an apatite layer after SBF dipping, do not directly bond to bone in vivo. Our data completely ruled out hydroxyapatite formation on the RVC foams in SBF. Nevertheless, the MSCs adhered perfectly well to the material in vitro and most importantly, the implants were completely integrated into the newly formed bone. Thus, this "non-bioactive" material as it would be defined with respect to its behaviour in SBF, demonstrated surprisingly good bioactivity in bone.

In contrast, material fragments in osteochondral defects were only observed two weeks post-implantation where they were found just beneath the implantation site, embedded in the spongy bone. Interestingly, several implant fragments were observed in direct contact with ectopic cartilage tissue formation at that early time point. Such cartilaginous nuclei can normally be found later after defect setting, in circumstances of incomplete cartilage regeneration, when a plug of fibrocartilage ends up closing the defect but does not convert into functional, hyaline cartilage. The ectopic cartilaginous formations detected in our samples might be the consequence of a previous association of mesenchymal stroma cells with the scaffold fibres, followed by their differentiation into chondrocytes, as proposed by Pulliainen et al. (2007) for poly-L-D-lactic acid (PLDLA) implants. In support of this hypothesis, we found that bone marrow-derived mesenchymal cells adhered easily to our foams in vitro and grew well in 3D cultures. At six and twelve weeks post-implantation, no more material fragments were detected at the implantation sites and the osteochondral defects were filled with fibrocartilage similarly as in the control animals.

Fibrocartilage is a dense connective tissue characterized by a high content of collagen fibres, rendering it more compact than for example loose connective tissue. For this reason and in accordance with the data assessed by Pulliainen et al. (2007) for their similarly hard PLDLA implants, soft connective tissues may adapt easier to the stiff nature of the RVC constructs. Thus, the implants may have been extruded out of the defect during the formation of fibrocartilage, a hypothesis supported by the fact that no more material fragments were found within the defect twelve weeks post-implantation. The PLDLA implants mentioned above had been driven out of the repair zone even when chondrocyte pre-seeded scaffolds were used and despite of a relatively thick layer of cartilage in that animal model. Our hypothesis of a mere mechanical but not biological incompatibility of Duocel ${ }^{\circledR}$ RVC with cartilage is further strengthened by two findings: Primary chondrocytes adhered perfectly well to the material in vitro. The fact that a moderate growth inhibition of 2D cultured MSCs was observed in the presence of RVC scaffolds, could point to a foreign body reaction in vivo. But most importantly, no giant cells at all and no further signs of inflammation were detected at the osteochondral defect sites although the implants had been extruded out.

Interestingly, the rabbit chondrocytes not only adhered easily but also exhibited all stages of adhesion to the RVC branches, from round to completely spread cells. Chondrocytes within the extracellular matrix of hyaline cartilage (Fig. 4b), but also for example in collagen or hydrogel matrices do not spread. In the round stage, chondrocytes hardly proliferate but maintain well their original phenotype (Kimura et al., 1984, Gründer et al., 2004). To expand the small samples that result from cartilage biopsies for tissue engineering and/or reimplantation purposes, the cells are usually cultured under 2D conditions, which implicates total cell spreading and a higher degree of proliferation but also a gradual loss of the differentiated phenotype. Depending on whether the cells had undergone serial passaging under 2D conditions or not, this phenotype may be partially or completely recovered by transferring the cells into an environment that prevents cell spreading (Martin et al., 1999). Thus, although our data indicate incompatibility of RVC with cartilage regeneration, it might be applied for in vitro chondrocyte expansion in $3 \mathrm{D}$. The combination of $3 \mathrm{D}$ culture conditions and partial chondrocyte spreading could favour cell proliferation without dedifferentiation or maintaining good redifferentiation capacity of the cells, a fact worthwhile further and detailed study.

We feel that attention should be drawn to the fact that in spite of the moderate inhibition of MSCs no signs of RVC cytotoxicity were observed in the 3D MSC cultures. Cell adhesion to $3 \mathrm{D}$ structures leads to a completely different response of the cytoskeleton compared to cell culture on plastic. Especially the actin fibres form part of a complex signalling network related with cell migration, survival and proliferation. Thus, we hypothesize that our seemingly adverse data of material cytotoxicity in MSCs are overcome by its favourable effects on the MSCs when cultured in 3D on the RVC branches.

As mentioned before, the published data about carbon material and carbon composite implants are diverse and range from reports about good compatibility, even in clinical situations, over some foreign body reactions (Aoki et al., 2009) to severe material rejection in patients with Proplast II mandibular implants (Lypka and Yamashita, 2007; Abramowicz et al., 2008). Importantly, our implants were not only integrated into the regenerating bone tissue, but even led to complete defect repair without application of any additional growth factor. In contrast, the carbon fibre sheets of Aoki et al. (2009), for example, led only to slight bone formation when BMP-2 was not added.

In vitro, during the cell loading procedure, excess medium was observed to pour out of the scaffolds on the opposite side, raising the suspicion of a considerable cell 
loss. Nonetheless, few cells were observed on the bottom of the culture dishes after loading and many adherent cells were detected on the Duocel ${ }^{\circledR}$ foam fibres after two weeks of culture. These cell populations may have been the result of a significant number of cell doublings in culture, but they also demonstrate that the material favours cell adhesion to a remarkable extent without any pre-treatment. Many studies are dedicated to the optimization of scaffold surfaces to promote cell adhesion, for example by coating with proteins (Yoo et al., 2005), specific cell adhesion molecule binding peptides (Yoon et al., 1994) or treatments to achieve more hydrophilic surfaces (Park et al., 2005). Our study revealed that Duocel ${ }^{\circledR}$ RVC supports cell adhesion of at least two different adherent cell types without the necessity of any surface manipulation.

Cell survival in $3 \mathrm{D}$ constructs is limited by oxygen and nutrient supply. Even highly porous scaffold structures that lack sufficient interconnectivity may limit mass transport and thus hinder cell migration into and/or survival inside their inner zones (Yang et al., 2001). The highly interconnective pores of our scaffolds favoured cell adhesion and survival even in their innermost zones and under steady state in vitro culture conditions during the test period of two weeks.

There is a lively interest in determining the optimum pore structure of materials such as calcium phosphate to facilitate different bone applications (Uchida et al., 1984; Chang et al., 2000; Chu et al. 2002). However, it is still a challenge to synthesize perfectly controlled scaffold geometries. Bohner and Baumgart (2007) found out that a pore diameter in the range of $200-800 \mu \mathrm{m}$ is optimal but that this optimum depends on the size and type of bone to be substituted. On the whole, most studies agree with a pore diameter in the range of 100-1000 $\mu \mathrm{m}$ (Uchida et al., 1984; Chang et al., 2000; Chu et al., 2002), the necessity of interconnected pores, and interconnections of more than about $50 \mu \mathrm{m}$ (Lu et al., 1999) to allow for tissue ingrowth and formation. The herein presented data demonstrate that the pore size of $200-400 \mu \mathrm{m}$ together with the high porous interconnectivity of the RVC foams used in this study was appropriate with respect to in vitro cell cultures and to our bone defect, as total defect regeneration was achieved.

\section{Conclusion}

The in vivo and in vitro results presented in this work clearly point out the potential of RVC foams as constructs for tissue engineering and regenerative applications. Because RVC foams are available within a wide range of pore sizes and offer an unusual porous interconnectivity, they could be easily adapted to the necessities of a variety of bone models and regenerative medical applications. In addition, their favourable properties for cell adhesion could give rise to their application for ex vivo cell expansion and/or tissue engineering and should be an issue for future investigation.

\section{Acknowledgements}

This work was supported by the Ministry of Science and Technology (MAT2008-02632/MAT to C.E. and Hospitén Holding (CI02320701). R.R. and M.K.P. were financed by the Motiva Project of FUNCIS, ACIISI. M.A. acknowledges the support from the Ramón y Cajal programme.

\section{References}

Abramowicz S, Dolwick MF, Lewis SB, Dolce C (2008) Temporomandibular joint reconstruction after failed Teflon-proplast implant: case report and literaturereview. Int J Oral Maxillofac Surg 37: 763-767.

Aoki K, Usui Y, Narita N, Ogiwara N, Iashigaki N, Nakamura K, Kato H, Sano K, Ogiwara N, Kametani K, Kim C, Taruta S, Kim YA, Endo M, Saito N (2009) A thin carbon-fiber web as a scaffold for bone-tissue regeneration. Small 5: 1540-1546.

Barber JC, Feaster F, Priour D (1980) The acceptance of a vitreous carbon alloplastic material, Proplast, in the rabbit eye. Invest Ophthalmol Vis Sci 19: 182-191.

Bohner M, Lemaitre J (2009) Can bioactivity be tested in vitro with SBF solution? Biomaterials 30: 2175-2179.

Bohner M, Baumgart F (2004) Theoretical model to determine the effects of geometrical factors on the resorption of calcium phosphate bone substitutes. Biomaterials 25: 3569-3582.

Borman JB, Brands WGB, Camilleri L, Cotrufo M, Daenen W, Gandjbakhch I, Infantes C, Khayat A, Laborde F, Pellegrini A, Piwnica A, Reichart B, Sharony R, Walesby R, Warembourg H (1998) Bicarbon valve - European multicenter clinical evaluation. European J Cardiothorac Surg 13: 685-693.

Chang B-S, Lee C-K, Hong K-S, Youn H-J, Ryu H-S, Chung S-S, ParkK-W (2000) Osteoconduction at porous hydroxyapatite with various pore configurations. Biomaterials 21: 1291-1298.

Chu T-MG, Orton DG, Hollister SJ, Feinberg SE, Halloran JW (2002) Mechanical and in vivo performance of hydroxyapatite implants with controlled architectures. Biomaterials 23: 1283-1293.

Dobson KR, Reading L, Haberey M, Marine X, Scutt A (1999) Centrifugal isolation of bone marrow from bone: an improved method for the recovery and quantitation of bone marrow osteoprogenitor cells from rat tibiae and femurae. Calcif Tissue Int 65: 411-413.

Friedrich JM, Ponce-de-Leon C, Reade GW, Walsh FC (2004) Reticulated vitreous carbon as an electrode material. J Electr Chem 561: 203-217.

Gründer T, Gaissmaer c, Fritz J, Stoop R, Hortschansky P, Mollenhauer J, and Aicher WK (2004) Bone morphogenic protein (BMP)-2 enhances the expression of type II collagen and aggrecan in chondrocytes embedded in alginate beads. OsteoArthritis and Cartilage 12: 559567.

Kent BL, Mutharasan R (1992) Cultivation of animal cells in a reticulated vitreous carbon foam. J Biotechnol 22: $311-328$. 
Kimura T, Yasui N, Ohsawa S, Ono K (1984) Chondrocytes embedded in collagen gels maintain cartilage phenotype during long-term cultures. Clin Orthop Rel Res 186: 231-239.

Kokubo T, Kushitani H, Sakka S, Kitsugi T, Yamamuro $\mathrm{T}$ (1990) Solutions able to reproduce in vivo surfacestructure changes in bioactive glass-ceramic A-W3. J Biom Mater Res 24: 721-734.

Komarasamy B, Vadivelu R, Bruce A, Kershaw C, Davison J (2006) Clinical and radiological outcome following total hip arthroplasty with an uncemented trabecular metal monoblock acetabular cup. Acta Orthop Belg 72: 320-325.

Lu JX, Flautre B, Anselme K, Hardouin P, Gallur A, Descamps M, Thierry B (1999) Role of interconnections in porous bioceramics on bone recolonization in vitro and in vivo. J Mater Sci Mater Med 10: 111-120.

Lypka M, Yamashita DD (2007) Exuberant foreign body giant cell reaction to a teflon/proplast temporomandibular joint implant: report of a case. J Oral Maxillofac Surg 65: 1680-1684.

Martin I, Vunjak-Novakovic G, Yang J, Langer R, Freed LE (1999) Mammalian chondrocytes expanded in the presence of fibroblast growth factor 2 maintain the ability to differentiate and regenerate three-dimensional cartilaginous tissue. Exp Cell Res 253: 681-688.

Misawa Y, Saito T, Konishi H, Oki S, Kaminishi Y, Sakano Y, Morita H, Aizawa K (2007) Clinical experience with Bicarbon heart valve prosthesis. J Cardiothorac Surg 25: 2-8.

Mulier M, Rys B, Moke L (2006) Hedrocel trabecular metal monoblock acetabular cups: mid-term results. Acta Orthop Belg 72: 326-331.

Nicholson P, Mulcahy D, Curtin B, McElwain JP (1998) Role of carbon fibre implants in osteochondral defects of the knee. Irish J Med Sci 167: 86-88.

Park GE, Pattison MA, Park K, Webster TJ (2005) Accelerated chondrocyte functions on $\mathrm{NaOH}$-treated PLGA scaffolds. Biomaterials 26: 3075-3082.

Parsons JR, Weiss AB, Schenk RS, Alexander H, Pavlisko F (1989) Long-term follow-up of achilles tendon repair with an absorbable polymer carbon fiber composite. Foot Ankle 9(4): 179-184.

Pulliainen O, Vasara AI, Hyttinen MM, Tiitu V, Valonen P, Kellomäki M, Jurvelin JS, Peterson L, Lindahl A, Kiviranta I, Lammi MJ (2007) Poly-L-D-lactic acid scaffold in the repair of porcine knee cartilage lesions. Tissue Eng 13: 1347-1355.

Qiu YS, Shahgaldi BF, Revell WJ, Heatley FW (2002) Evaluation of Gateshead carbon fibre rod as an implant material for repair of osteochondral defects: A morphological and mechanical study in the rabbit knee. Biomaterials 23: 3943-3955.

Rogulski Z, Lewdorowicz W, Tokarz W, Czerwinski A (2004) Applications of reticulated vitreous carbon (RVC) in the electrochemical power sources. J Pol Chem 78: 1357 1370 .

Shah S, Rhatigan M, Sampath R, Yeoman C, Sunderland S, Brammer R, Leatherbarrow B (1995) Use of Proplast II as a subperiosteal implant for the correction of anophthalmic enophthalmos. British J Ophthalmol 79: 830-833.

Sumita O (1985) Applications of reticulated vitreous carbon. Kagaku Sochi 27:

60-69.

Turgut G, Eksilioglu A, Gencay N, Gonen E, Hekim N, Yardim MF, Sakiz D, Ekinci E (2008) Pore structure engineering for carbon foams as possible bone implant material. J Biomed Mater Res 85A: 588-596.

Uchida A, Nade SM, McCartney EF, Ching W (1984) The use of ceramics for bone replacement. A comparative study of three different porous ceramics. J Bone Joint Surg Br 66: 269-275.

Visuri T, Kiviluoto O, Eskelin M (1991) Carbon fiber for repair of the rotator cuff. A 4-year follow-up of 14 cases. Acta Orthop Scand 62: 356-369.

Wickham MG, Cleveland PH, Binder PS, Akers PH (1983) Growth of cultured corneal endothelial cells onto a vitreous carbon matrix. Ophthalmic Res 15: 116-120.

Wigfield C, Robertson J, Gill S, Nelson R (2003) Clinical experience with porous tantalum cervical interbody implants in a prospective randomized controlled trial. Br J Neurosurg 17: 418-425.

Yang S, Leong KF, Du Z, Chua CK (2001) The design of scaffolds for use in tissue engineering. I. Traditional factors. Tissue Eng 7: 679-689.

Yoo HS, Lee EA, Yoon JJ, Park TG (2005) Hyaluronic acid modified biodegradable scaffolds for cartilage tissue engineering. Biomaterials 26: 1925-1933.

Yoon JJ, Song SH, Lee DS, Park TG (1994) Immobilization of cell adhesive RGD peptide onto the surface of highly porous biodegradable polymer scaffolds fabricated by a gas foaming/salt leaching method. Biomaterials 25: 5613-5620.

Zardiackas LD, Parsell DE, Dillon LD, Mitchell DW, Nunnery LA, Poggie R (2001) Structure metallurgy, and mechanical properties of a porous tantalum foam. J Biomed Mater Res (Appl Biomater) 58: 180-187.

\section{Discussion with Reviewers}

Reviewer I: What happens to the foam that does not appear in the images (Fig. 8)?. Is this a technical artefact?

Authors: We think that this phenomenon is not a technical artefact; in fact there were small traces of RVC foams in some of the sections.

Reviewer I: If there is no inflammatory or foreign body reaction, where is the RVC foam?

Authors: As mentioned in the discussion, we think that the scaffold was extruded from the osteochondral defect and probably has been driven out of the bone into the surrounding soft tissue. Thus, we did not locate it within the specimen.

Reviewer II: Please comment on the cytotoxicity study. A control or a comparator material would be useful to understand if this level of cytotoxicity could have detrimental effects. 
Authors: The issue of determining the toxicity of a material or compound is complex. We have carried out the cytotoxicity study the way it is frequently done with materials and always with soluble compounds: exposing the plastic-adhered cells to the material and comparing the results with those obtained from "untreated", unexposed control cells.

However, there are two points that most likely prevent these results from being directly transferable to the in vivo situation:

1. Implanted materials will be exposed to the physiological environment including blood and lymphatic drainage which reduce local cell exposure to possible harming agents.

2. Cells grown in a three dimensional (3D) environment are subjected to distinct tensile forces than on culture plastic (2D). These forces are transmitted to the cytoskeleton and from there to a network of signalling pathways, some of them related to cell survival and/or metabolism. Hence, the cells may respond differently to the same agent depending on the substrate they are cultured on. Actually, our microphotographs demonstrate that both cell types looked fine when cultured on the carbon foams.

On the other hand, it has to be mentioned that mesenchymal stem cells, similarly as other blood derived cells, are especially delicate with respect to cytotoxicity, at least when grown in vitro. MSCs or lymphocytes, for example, cannot be grown in copper-coated $\mathrm{CO}_{2}$ incubators, where other cell types do well.

In our laboratory we are used to obtaining growth reduction with bone marrow derived rat MSCs, even when exposing them to well-established materials as for example tricalcium phosphate $(72 \%)$ or pure chitosan $(84 \%)$ compared to non-exposed control cells (100\%). The only material that did not affect these cells in 2D conditions until now was PLA (polylactide). So once again, we think that the excellent outcome with the material in $3 \mathrm{D}$ cultures and the total absence of inflammation and foreign body reactions in vivo override the concern that the observed level of cytotoxicity in 2D cultures of MSCs could have detrimental effects.

Reviewer III: Do you have evidence that the bone defect is critical in size?

Authors: The bone defect is not critical in size. However, our results demonstrate the validity of our defect model to evaluate degree and duration of tissue regeneration with that material. Differences between empty controls and implanted defects were apparent at all three sampling time points, ending up in several ossification foci and loose connective tissue in the controls versus complete bone regeneration in the implanted samples after 12 weeks.

Reviewer III: The cytotoxicity data shows a drop in viability for rat MSCs. Is this a concern given the permanent nature of your scaffold?

Authors: Mesenchymal stem cells, similarly to other blood derived cells, are especially delicate with respect to cytotoxicity, at least when grown on cell culture plastic. In our laboratory we are used to obtain growth reduction with bone marrow derived mesenchymal stroma cells (and much less with primary cells from other tissues) when exposing them to well-established bio-materials.

Cells grown in a three dimensional (3D) environment are subjected to the induction of cytoskeleton transmitted signalling pathways, some of them related to cell survival and/or metabolism, which change their status as a whole. They may respond differently to the same agent depending on the substrate they are cultured on. Actually, our microphotographs demonstrate that both cell types looked fine when cultured on the carbon foams.

On the other hand, the implanted materials were exposed to the physiological environment including blood and lymphatic drainage which reduce local cell exposure to possibly harming agents. The total absence of inflammation and foreign body reaction in vivo indicate that the observed level of cytotoxicity in 2D cultures of MSCs does not result in detrimental effects in vivo, at least during a period of 12 weeks. Moreover, the material allowed the MSCs to differentiate and go through the whole cascade of bone formation. 